\title{
Schwere Hypoglykämien erhöhen die Mortalität
}

\author{
Eine prospektive Studie liefert starke Hinweise dafür, dass schwere Hypoglykämien das Sterberisiko \\ von Typ-2-Diabetikern erhöhen. Vor allem sollte das Gefäßrisiko der Betroffenen ermittelt werden.
}

_ Einige Studien konnten bereits zeigen, dass schwere Hypoglykämien ( $\mathrm{SH}$ ) bei Typ-2-Diabetikern mit einem erhöhten Sterberisiko verknüpft sind [Goto A et al. BMJ. 2013;347:f4533; Zoungas S et al. N Engl J Med. 2010;363:1410-8]. Für eine prospektive Kohortenstudie in den Jahren 2000-2010 wurden 1.260 Patienten im Alter von 25-75 Jahren ohne vorherige $\mathrm{SH}$ einbezogen. Sie wurden im Mittel 10,4 Jahre lang beobachtet.

Von den 906 Patienten, die das Follow-up abschlossen, wurden 9,4\% wenigstens einmal wegen einer $\mathrm{SH}$ in einer Notaufnahme oder stationär behandelt. 86 Patienten (9,5\%) starben, davon 21 wegen eines kardiovaskulären Ereignisses. $\mathrm{SH}$ waren in einer multivariaten Analyse mit einem erhöhten Risiko für Tod insgesamt (Hazard Ratio 2,64, p < $0,003)$ wie auch für Tod mit kardiovas- kulärer Ursache (Hazard Ratio 6,34, p < 0,002) assoziiert.

Berücksichtigt wurden dabei Geschlecht, Dauer der Diabeteserkrankung, Hypertonie, mittlere $\mathrm{HbA}_{1 \mathrm{c}}$-Werte, diabetische Nephropathie, Hyperlipidämie und Insulingebrauch. Die SHAnamnese erwies sich somit als signifikanter prognostischer Faktor für einen kardiovaskulär bedingten Tod.

- Cha SA, Yun JS, Lim TS et al. Severe hypoglycemia and cardiovascular or all-cause mortality in patients with type 2 diabetes. Diabetes Metab J. 2016;40:202-10

\section{KOMMENTAR}

Die prospektive Langzeitstudie konnte zeigen, dass schwere Hypoglykämie-Ereignisse bei Typ-2-Diabetes signifikant mit einer erhöhten kardiovaskulären und Gesamtmortalität assoziiert waren. Die Resultate waren unabhängig von Blutzuckereinstellung, Dia- betesdauer, Alter, diabetischer Nephropathie, Gebrauch von Insulin oder kardiovaskuIären Erkrankungen. Kardiovaskuläre Ereignisse waren für $24,4 \%$ der Todesfälle verantwortlich. Die Stärken der Studie sind das prospektive Design, die lange Beobachtungszeit und die klare Definition von objektivierten hypoglykämischen Episoden.

Die Autoren legen nahe, dass das Auftreten von SH ein unabhängiger Risikofaktor für spätere kardiovaskuläre Ereignisse ist - und die Patienten daher entsprechend auf bisher unbekannte kardiovaskuläre Risiken untersucht werden sollten. Hinsichtlich der kausalen pathogenetischen Mechanismen kommen wohl erhöhte Sympathikusaktivierung, Entzündungen, endotheliale Dysfunktion und veränderte Gerinnung infrage [Benedis $R$ et al. Diabetes Care. 2014;37:3301-8; Stahn A et al. Diabetes Care. 2014;37:516-20].

Prof. Dr. med. E. Fritschka

\section{Trommelschlegelfinger können sich zurückbilden}

Eine 59-jährige Frau mit einer Raucheranamnese von 60 Packungsjahren stellte sich wegen zunehmender Kurzatmigkeit und Trommelschlegel-
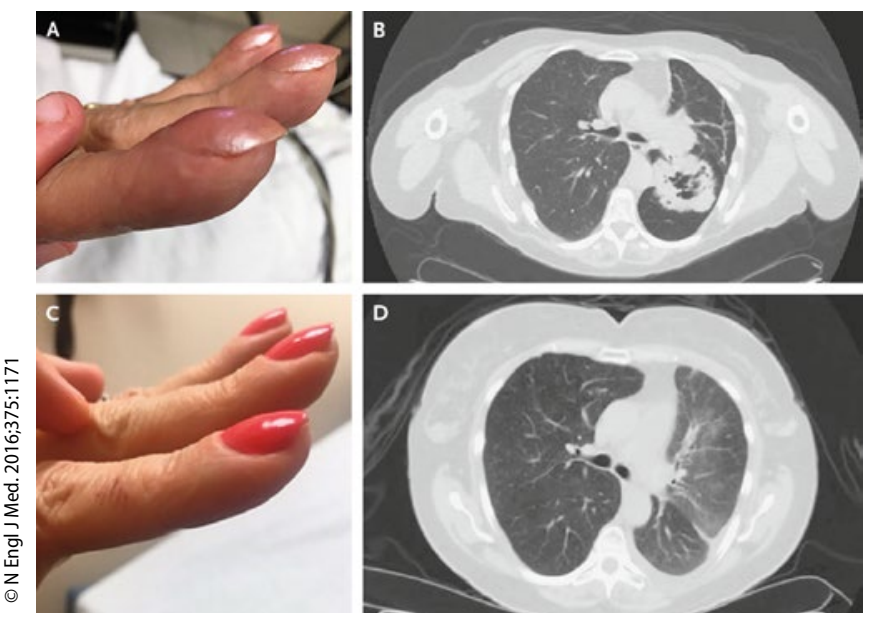

Trommelschlegelfinger (A), Raumforderung im Lungenunterlappen links (B), Zustand sechs Monate später (C, D). fingern vor, die sich im Lauf des letzten Jahres ausgebildet hatten (Abb. A). Im Thorax-CT zeigte sich eine große Raumforderung mit Kavernenbildung im linken Unterlappen (Abb. B). Bioptisch ergab sich ein undifferenziertes, nicht-kleinzelliges Karzinom mit positiver Immunhistochemie für Tumorprotein 63 und Cytokeratin 5/6, vereinbar mit der Diagnose eines schlecht differenzierten Plattenepithelkarzinoms der Lunge. Die Patientin wurde mit Carboplatin und Paclitaxel sowie Bestrahlung behandelt und in eine Studie zur Immuntherapie mit einem Hemmer des programmierten Zelltods (PD-L1) als Erhaltungstherapie einbezogen.

Sechs Monate nach Diagnosestellung hatten sich die Trommelschlegelfinger der Patientin zurückgebildet (Abb. C). Noch mehr dürfte sie sich darüber gefreut haben, dass ihre Kurzatmigkeit verschwunden war. Eine CTNachkontrolle zeigte eine deutliche Größenabnahme des Tumors (Abb. D).

Neu aufgetretene Trommelschlegelfinger sollten den Verdacht auf ein Bronchialkarzinom lenken, wenngleich auch andere Lungenerkrankungen dieses Phänomen auslösen können. Früher hat man die Rückbildung von Trommelschlegelfingern kaum gesehen, da Patienten mit Bronchialkarzinom in der Regel nicht so lange überlebten.

Prof. Dr. med. H. S. FüeßI

- Ciment AJ, Ciment L (airciment@aol.com): Regression of clubbing after treatment of lung cancer. N Engl J Med. 2016;375:1171 